\title{
Nutritional aspects and cardiovascular risk in systemic lupus erythematosus
}

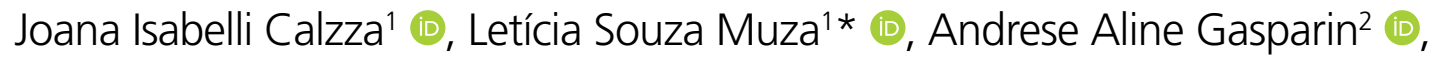 \\ Ricardo Machado Xavier² (1), Odirlei André Monticielo²
}

\begin{abstract}
SUMMARY
OBJECTIVE: Patients with systemic lupus erythematosus present with a higher number of classic risk factors for coronary diseases and a higher prevalence of metabolic syndrome resulting from the disease itself. To evaluate the nutritional indicators of the cardiovascular risk of patients with systemic lupus erythematosus by analyzing eating habits, anthropometry, laboratory data, and disease activity and to describe the prevalence of patients fulfilling the criteria for metabolic syndrome.

METHODS: Anthropometric measurements including waist circumference, food recall, and laboratory tests.

RESULTS: The population presented an insufficient daily intake of micronutrients. Anthropometry revealed that $37.5 \%$ of the patients were classified with degree II obesity by body mass index and $76.8 \%$ by abdominal obesity. Regarding metabolic syndrome, 18 patients (16\%) fulfilled the diagnostic criteria.

CONCLUSIONS: Individuals with systemic lupus erythematosus presented with increased risk factors, as determined using anthropometric measurements and laboratory tests, for cardiovascular disease, indicating the need for nutritional guidance in this population to reduce cardiovascular risk, increase the quality of life, and increase survival of these patients.

KEYWORDS: Nutrition. Cardiovascular system. Systemic lupus erythematosus.
\end{abstract}

\section{INTRODUCTION}

Systemic lupus erythematosus (SLE) is an autoimmune, chronic, multisystemic, relapsing-remitting autoimmune disease characterized by mucocutaneous lesions, hematological changes, and inflammation in joints, kidneys, serous membranes, and other organs associated with the presence of self-reactive antibodies and occurring predominantly in women. The Systemic Lupus International Collaborating Clinics Damage Index (SLICC) and the Systemic Lupus Erythematosus Disease Activity Index (SLEDAI) are used to evaluate chronicity and disease activity. Cardiovascular disease (CVD) is an important cause of premature death in patients with $\mathrm{SLE}^{1}$. Compared to the general population, patients with SLE have a greater number of classic risk factors for CVD, such as obesity, dyslipidemia, and metabolic syndrome (MS), which are partly associated with nutritional aspects ${ }^{1,2}$.

MS causes a 1.5-fold increase in overall mortality and a 2.5fold increase in the risk for $\mathrm{CVD}^{1,2}$, with a prevalence ranging from 20 to $28.4 \%$ in patients with $S_{L E}^{2,3}$. The prevalence of dyslipidemia in patients with SLE ranges from 36\% at diagnosis to $60 \%$ or more after 3 years ${ }^{4,5}$. Its occurrence is specifically related to inflammatory disease activity and corticosteroid use $\mathrm{e}^{4}$. The treatment of systemic inflammation with corticosteroids, in addition to disordered eating habits and reduced physical

\footnotetext{
'Universidade Federal do Rio Grande do Sul, Departamento de Nutrição - Porto Alegre (RS), Brazil.

${ }^{2}$ Hospital de Clínicas de Porto Alegre, Serviço de Reumatologia - Porto Alegre (RS), Brazil.

*Corresponding author: leticia.souza@ufrgs.br

Conflicts of interest: the authors declare there is no conflicts of interest. Funding: none.

Received on January 25, 2021. Accepted on February 06, 2021.
} 
activity, may increase the predisposition of accumulating body fat and of developing a coronary disease in patients with SLE. Abdominal obesity is commonly associated with the development of atheroma and $\mathrm{CVD}^{6}$.

Regarding nutritional aspects in SLE, a review article showed that patients with SLE can benefit from a nutritionally balanced diet to maintain ideal body weight and from effective calorie control to avoid insulin resistance, by increasing high-density lipoprotein (HDL) and decreasing triglyceride levels ${ }^{7}$.

In addition to dietary characteristics, the identification of anthropometric indicators helps in the early identification of nutritional aspects that increase the risk for CVD. The objective of this study was to evaluate the nutritional profile of patients with SLE by analyzing food intake, anthropometric indicators, and the association of disease activity and cardiovascular risk.

\section{METHODS}

\section{Study design and population}

This is a cross-sectional study with 112 patients diagnosed with SLE according to the diagnostic classification criteria described in 1982 and updated in $1997^{\circ}$. The studied population included random patients treated in an outpatient clinic created specifically for patients with SLE at the Rheumatology Service of the Hospital de Clínicas de Porto Alegre, Rio Grande do Sul, Brazil. The data were collected from May 2015 to February 2018. During routine consultations, patients were invited to participate in a study on the nutritional aspects of SLE to support the creation of future nutritional clinical strategies as treatment. All patients who agreed to participate signed an informed consent form. The exclusion criteria were the presence of metabolic modifying factors that could interfere with the nutritional evaluation, such as acquired immunodeficiency syndrome (AIDS) and being pregnant or breastfeeding at the time of evaluation.

\section{Nutritional profile evaluation}

The evaluation consisted of anthropometric, dietary, and laboratory measurements. Anthropometric data were measured according to the standards of the World Health Organization?. Body mass index (BMI) was calculated using the formula $\mathrm{BMI}=$ weight $/$ height $^{2}$. Body fat percentage (BFP) was determined by body density using the equations proposed by Siri in $1961^{10}$. Abdominal obesity was determined by waist circumference (WC) using the cutoff points of $\geq 90 \mathrm{~cm}$ for men and $\geq 80$ $\mathrm{cm}$ for women ${ }^{11}$. An anthropometric variable called metabolic risk (MR) was created for the present study and defined as the group of participants concomitantly presenting all the anthropometric classifications of obesity.

Dietary indicators were evaluated using 24-hour dietary recall, which was subsequently processed in the DietWin Nutritional Analysis software version $2.0^{\circledR}$.

The criteria for MS classification were based on the identification of three or more factors in the same person: abdominal obesity ( $\geq 90 \mathrm{~cm}$ in men and $\geq 80 \mathrm{~cm}$ in women); HDLcholesterol $<40 \mathrm{mg} / \mathrm{dL}$ in men and $<50 \mathrm{mg} / \mathrm{dL}$ in women; triglycerides $\geq 150 \mathrm{mg} / \mathrm{dL}$; systemic blood pressure $\geq 135 / 85$ $\mathrm{mmHg}$ or use of antihypertensive drugs; and, fasting glucose $\geq 110 \mathrm{mg} / \mathrm{dL}^{12}$.

\section{Laboratory measurements}

The results of the following tests were collected directly from the patients' medical records: complete blood count; creatinine, glucose, total cholesterol, HDL, low-density protein (LDL), triglycerides, anti-double-stranded DNA, complement (C3 and C4) levels; and qualitative urine test.

\section{Statistical analysis}

The Statistical Package for Social Sciences software (SPSS) version 1.8.0 was used in the statistical analyses. The Fisher's exact test was used to determine the association between two or more qualitative random variables. The Pearson's Chi-square test was used to analyze probability in categorical data for unpaired samples. The Mann-Whitney test was used for independent samples. A $\mathrm{p}<0.05$ was considered statistically significant.

\section{Ethical aspect}

The identity of the study participants was kept confidential and all participants signed an informed consent form. The study was approved by the Research Ethics Committee of the Federal University of Rio Grande do Sul under Certificado de Apresentação para Apreciação Ética (CAAE) no. 4338331 4.2.0000.5347.

\section{RESULTS}

The study population consisted of 112 patients, 106 (94.6\%) of whom were women. Age ranged from 40 to 61 years for men and from 28 to 80 years for women. A total of 77 patients $(68.8 \%)$ patients presented a score of 0 in the SLICC, similar to scores for disease activity, with 73 patients $(62.5 \%)$ presenting a score of 0 in the SLEDAI. The $25^{\text {th }}$ to $75^{\text {th }}$ percentiles of the medians were 0 to 1 for the SLICC and SLEDAI, as shown in Table 1. 
Anthropometric data showed an obesity classification grade II according to BMI in 42 patients $(37.5 \%)$, whereas $26(23.2 \%)$ had grade I obesity, and 35 (31.3\%) were overweight. The evaluation of adiposity according to BFP showed 48 patients (42.9\%) and 25 patients $(22.3 \%)$ being classified as very high and high risk, respectively, totaling 73 patients (65.2\%) classified as having high body fat. Abdominal obesity was present in 86 patients $(76.8 \%)$, as shown in Table 2. Regarding eating habits, there was an insufficient daily intake of micronutrients, mainly of potassium, copper, boron, and

Table 1. Descriptive data of the study population.

\begin{tabular}{|c|c|c|}
\hline & $\mathrm{n}$ & $\%$ \\
\hline \multicolumn{3}{|l|}{ Sex } \\
\hline Female & 106 & 94.6 \\
\hline Male & 6 & 5,4 \\
\hline Total & 112 & 100 \\
\hline \multicolumn{3}{|c|}{ Classification } \\
\hline \multicolumn{3}{|l|}{ SLICC } \\
\hline 0 & 77 & 68.8 \\
\hline 1 & 11 & 9.8 \\
\hline 2 & 5 & 4.5 \\
\hline 3 & 12 & 10.7 \\
\hline 4 & 3 & 2.7 \\
\hline 5 & 2 & 1.8 \\
\hline 6 & 1 & 0.9 \\
\hline 8 & 1 & 0.9 \\
\hline Total & 112 & 100 \\
\hline \multicolumn{3}{|l|}{ SLEDAI } \\
\hline 0 & 70 & 62.8 \\
\hline 1 & 5 & 4.5 \\
\hline 2 & 11 & 9.8 \\
\hline 3 & 2 & 1.8 \\
\hline 4 & 15 & 12.4 \\
\hline 5 & 2 & 1.8 \\
\hline 6 & 4 & 3.6 \\
\hline 8 & 2 & 1.8 \\
\hline 9 & 1 & 0.9 \\
\hline Total & 112 & 100 \\
\hline
\end{tabular}

SLICC: Systemic Lupus International Collaborating Clinics Damage Index, SLEDAl: Systemic Lupus Erythematosus Disease Activity Index. vitamin $\mathrm{B} 1$, with $100 \%$ of the participants consuming less than the daily quantity recommended.

MR was present in 58 patients (51.8\%) who showed an association between MR and low dietary intake of vitamin B9, choline, and sulfur, as shown in Table 3. Regarding MS, 18 patients $(16 \%)$ met the classification criteria. Laboratory tests showed that 31 patients (27.7\%) had a high LDL level, $64(57.1 \%)$ had a moderate or high-risk HDL level, seven (6.3\%) had a high-risk cholesterol level, and 10 (8.9\%) had triglyceride levels above the borderline cutoff.

Table 2. Anthropometric data of the study population.

\begin{tabular}{|c|c|c|}
\hline & $n$ & $\%$ \\
\hline \multicolumn{3}{|l|}{ BMI } \\
\hline Obesity II & 42 & 37.5 \\
\hline Obesity I & 26 & 23.2 \\
\hline Overweight & 35 & 31.3 \\
\hline Normal weight & 7 & 6.3 \\
\hline Low weight & 2 & 1.8 \\
\hline Total & 112 & 100 \\
\hline \multicolumn{3}{|l|}{ BFP } \\
\hline Very low & 3 & 2.7 \\
\hline Low & 17 & 15.2 \\
\hline Ideal & 19 & 17 \\
\hline High & 25 & 22.3 \\
\hline Very high & 48 & 42.9 \\
\hline Total & 112 & 100 \\
\hline \multicolumn{3}{|l|}{ WC } \\
\hline High & 86 & 76.8 \\
\hline Normal & 26 & 23.2 \\
\hline Total & 112 & 100 \\
\hline
\end{tabular}

BMI: body mass index, BFP: body fat percentage, WC: waist circumference.

Table 3. Association of metabolic risk and insufficient micronutrient intake.

\begin{tabular}{l|c|c} 
Micronutrients & $\mathrm{n}(\%)$ & $\mathrm{p}$ \\
\hline Choline & $49(84.5 \%)^{\mathrm{a}}$ & 0.048 \\
\hline Sulfur & $56(96.6 \%)^{\mathrm{b}}$ & 0.047 \\
\hline Vitamin B9 & $45(77.6 \%)^{\mathrm{a}}$ & 0.023 \\
\hline
\end{tabular}

${ }^{a}$ Chi-square and continuity correction tests; ${ }^{\text {b}}$ Chi-square and Fisher's exact tests.

Metabolic risk: concomitant classifications for obesity including body mass index, waist circumference, and percent body fat percentage. 


\section{DISCUSSION}

The patients in this study had low SLICC and SLEDAI scores, which indicates little accumulated damage and controlled disease at the time of evaluation, possibly indicating more controlled disease in this population ${ }^{1}$. The anthropometric evaluation showed a high prevalence of high BMI, corroborating the literature. Some studies show a high prevalence of obesity and suggest that obesity is associated with disease activity ${ }^{13,14}$. There was a high BFP in $62.5 \%$ of the patients when highand very-high-risk classifications were grouped. Some studies had similar results despite the use of other body adiposity measurement techniques. Borges et al. ${ }^{15}$ used bioimpedance and reported that $59.2 \%$ of the study population had a BFP above the recommended range.

This study showed excess abdominal fat according to WC in 86 patients (76.8\%), which represents a risk for CVD. A cohort study reported a high prevalence of abdominal obesity and a high incidence of cardiovascular risk in the SLE population ${ }^{6}$. Hormonal factors may be associated with increased weight and body fat in women with SLE, which were not addressed in the present study. However, they may be a topic for future research, as Li et al. ${ }^{16}$ stated that menopause was associated with increased BMI and abdominal obesity in patients with SLE. The literature emphasizes the association between abdominal obesity and CVD in the general population ${ }^{17}$. An experimental study on SLE showed that a high-fat diet was associated with visceral obesity ${ }^{18}$.

Regarding eating habits, although this study was limited to only one type of food survey, insufficient consumption of micronutrients was identified. Similar results were observed in the Mexican SLE population, regarding both the insufficient consumption of micronutrients and the excess weight ${ }^{14}$.

In another study, low fruit and vegetable consumption and insufficient dietary calcium and iron intake were observed ${ }^{15}$. The literature reports low micronutrient intake in patients with SLE and its possible association with atherosclerosis in this population ${ }^{19}$. The present study found an association of insufficient intake of some micronutrients with MR; i.e., an insufficient intake of folic acid (vitamin B9), choline, and sulfur was associated with the grouping of anthropometric indicators for obesity.

To date, there are no studies in the literature in the SLE population reporting similar results, possibly owing to the diversity of methodologies, although a study demonstrated that a dietary folic acid deficiency in SLE may be associated with atherosclerosis ${ }^{19}$, which in the present study was associated with MR. High micronutrient intake was reported as protective against excess weight in the Mexican SLE population ${ }^{14}$.
General population studies show micronutrient deficiencies in obese people, mainly of fat-soluble vitamins, B vitamins, and folic acid ${ }^{20}$. An experimental study found an association of choline deficiency with obesity ${ }^{21}$.

In the present study, 18 participants (16\%) met the classification criteria for MS. A meta-analysis study reported an MS prevalence of $26 \%$ in patients with SLE ${ }^{22}$. Regarding the lipid profile of this population, there was a predominance of results above the normal range, and the literature shows that patients with SLE often present changed plasma lipids and decreased HDL-cholesterol levels $s^{4,5}$. Dietary intake of saturated fats is one of the factors associated with an increased lipid profile and can play an important role in increasing these levels, and there is evidence of excessive oil and fat consumption by patients with SLE ${ }^{14,23}$. In a study with 170 patients $^{15}, 142(83.8 \%)$ consumed excess fat according to a food frequency questionnaire not used in the present study. The present study shows the importance of controlling risk factors through nutritional monitoring, preventing overweight, and decreasing anthropometric values, especially WC, which is the most associated with CVD, in addition to controlling adequate nutrient consumption. Nutritional profile factors can be modified in this population, indicating that risk factors can be controlled and/or treated with early nutritional clinical care, thus improving their metabolic complication outcomes.

\section{CONCLUSIONS}

Patients with SLE had a nutritional profile that increases CVD risk factors, which could possibly be reduced with clinical nutritional monitoring.

\section{AUTHORS' CONTRIBUTIONS}

JIC: Conceptualization, Data curation, Formal analysis, Investigation, Methodology, Software, Validation, Writing original draft. LSM: Conceptualization, Data curation, Formal analysis, Investigation, Methodology, Project administration, Software, Supervision, Visualization, Writing - original draft, Writing - review \& editing. AAG: Conceptualization, Data curation, Methodology, Supervision, Writing - review \& editing. RMX: Conceptualization, Data curation, Formal analysis, Investigation, Methodology, Project administration; Validation; Visualization; Writing - review \& editing. OAM: Conceptualization, Data curation, Formal analysis, Investigation, Methodology, Project administration, Supervision, Validation, Visualization, Writing - review $\&$ editing. 


\section{REFERENCES}

1. Kostopoulou M, Nikolopoulos D, Parodis I, Bertsias G. Cardiovascular disease in systemic lupus erythematosus: recent data on epidemiology, risk factors and prevention. Curr Vasc Pharmacol. 2020;18(6):549-65. https://doi.org/10.2174/157 0161118666191227101636

2. Medina $G$, Vera-Lastra $O$, Peralta-Amaro $A L$, JiménezArellano MP, Saavedra MA, Cruz-Domínguez MP, et al. Metabolic syndrome, autoimmunity and rheumatic diseases. Pharmacol Res. 2018;133:277-88. https://doi.org/10.1016/j. phrs.2018.01.009

3. Mok CC, Tse SM, Chan KL, Ho LY. Effect of the metabolic syndrome on organ damage and mortality in patients with systemic lupus erythematosus: a longitudinal analysis. Clin Exp Rheumatol. 2018;36(3):389-95. PMID: 29148424

4. Szabó MZ, Szodoray P, Kiss E. Dyslipidemia in systemic lupus erythematosus. Immunol Res. 2017;65(2):543-50. https://doi. org/10.1007/s12026-016-8892-9

5. Atta AM, Silva JPCG, Santiago MB, Oliveira IS, Oliveira RC, Sousa Atta MLB. Clinical and laboratory aspects of dyslipidemia in Brazilian women with systemic lupus erythematosus. Clin Rheumat. 2018;37(6):1539-46. https://doi.org/10.1007/ s10067-018-4051-0

6. Monção CSA, Martins LN, Penteado MPS, Reis RCP, Santos FMM, Lanna CCD, et al. Incidence of cardiovascular risk factors in female patients with systemic lupus erythematosus: a 3-year follow-up cohort. Lupus. 2018;27(11):1790-98. https://doi. org/10.1177/0961203318790676

7. Klack K, Bonfa E, Borba Neto EF. Diet and nutritional aspects in systemic lupus erythematosus. Rev Bras Reumatol. 2012;52(3):384-408. PMID: 22641593

8. Hochberg MC. Updating the American College of Rheumatology revised criteria for the classification of systemic lupus erythematosus. Arthritis Rheum. 1997;40(9):1725. https:// doi.org/10.1002/art.1780400928

9. World Health Organization. Physical status: the use and interpretation of anthropometry. Report of a WHO expert committee. Geneva: World Health Organization; 1995.

10. Siri WE. Body composition from fluid spaces and density. In: Brozec J, Henschel A (eds). Techniques for measuring body composition. Washington: National Academy of Science; 1961. p. 223-44.

11. Alberti KGMM, Zimmet $P$, Shaw J. Metabolic syndrome--a new world-wide definition. A consensus statement from the international diabetes federation. Diabet Med. 2006;23(5):46980. https://doi.org/10.1111/j.1464-5491.2006.01858.x

12. Alberti KGMM, Eckel RH, Grundy SM, Zimmet PZ, Cleeman J, Donato KA, et al. Harmonizing the metabolic syndrome: a joint interim statement of the International Diabetes Federation Task Force on Epidemiology and Prevention; National Heart, Lung, and Blood Institute; American Heart Association; World Heart Federation; International Atherosclerosis Society; and International Association for the Study of Obesity. Circulation. 2009;120(16):1640-5. https://doi.org/10.1161/ CIRCULATIONAHA.109.192644

13. Patterson SL, Schmajuk G, Jafri K, Yazdany J, Katz P. Obesity independently associates with worse patient-reported outcomes in women with systemic lupus erythematosus. Arthritis Care Res (Hoboken). 2019;71(1):126-33. https://doi.org/10.1002/ acr.23576

14. Meza-Meza MR, Vizmanos-Lamotte B, Muñoz-Valle JF, ParraRojas I, Garaulet M, Campos-López B, et al. Relationship of excess weight with clinical activity and dietary intake deficiencies in systemic lupus erythematosus patients. Nutrients. 2019;11(11):2683. https://doi.org/10.3390/nu11112683

15. Borges MC, dos Santos FMM, Telles RW, Lanna CCD, Correia MITD. Nutritional status and food intake in patients with systemic lupus erythematosus. Nutrition. 2012;28(11-12):1098103. https://doi.org/10.1016/j.nut.2012.01.015

16. Li Z, Shang J, Zeng S, Wu H, Zhou Y, Xu H. Altered body composition and increased visceral adipose tissue in premenopausal and late postmenopausal patients with SLE. Clin Rheumatol. 2019;38(11):3117-27. https://doi.org/10.1007/ s10067-019-04701-3

17. Barroso TA, Marins LB, Alves R, Gonçalves ACS, Barroso SG, Rocha GS. Association of central obesity with the incidence of cardiovascular diseases and risk factors. Int J Cardiovasc Sci. 2017;30(5):416-24. https://doi.org/10.5935/23594802.20170073

18. Gilbert EL, Ryan MJ. High dietary fat promotes visceral obesity and impaired endothelial function in female mice with systemic lupus erythematosus. Gend Med. 2011;8(2):150-5. https:// doi.org/10.1016/j.genm.2011.03.006

19. Lourdudoss C, Elkan AC, Hafström I, Jogestrand T, Gustafsson $T$, van Vollenhoven $R$, et al. Dietary micronutrient intake and atherosclerosis in systemic lupus erythematosus. Lupus. 2016;(14):1602-9. https://doi.org/10.1177/0961203316655211

20. Astrup A, Bügel $S$. Overfed but undernourished: recognizing nutritional inadequacies/deficiencies in patients with overweight or obesity. Int J Obes (Lond.). 2019;43(2):219-32. https://doi. org/10.1038/s41366-018-0143-9

21. Maswoswe SM, Tremblay GC. Biosynthesis of hippurate, urea and pyrimidines in the fatty liver: studies with rats fed orotic acid or a diet deficient in choline and inositol, and with genetically obese (Zucker) rats. J Nutr. 1989;119(2):273-9. https://doi.org/10.1093/jn/119.2.273

22. Sun C, Qin W, Zhang YH, Wu Y, Li Q, Liu M, et al. Prevalence and risk of metabolic syndrome in patients with systemic lupus erythematosus: a meta-analysis. Int J Rheum Dis. 2017;20(8):917-28. https://doi.org/10.1111/1756-185X.13153

23. achet JC, Borba Neto EF. Dyslipidaemia and atherosclerosis in systemic lupus erythematosus. Rev Bras Reumatol. 2003;43(5):324-8. 